\title{
Structural and Electrical Properties of $\mathrm{Ca}^{2+}$ Doped $\mathrm{LaFeO}_{3}$ : The Effect of A-site Cation Size Mismatch
}

\author{
Ali Ekber Irmak \\ Physics Department \\ Faculty of Science \\ Mugla Sitki Kocman University \\ Mugla, Turkey \\ ekberirmak@gmail.com
}

\begin{abstract}
In this study, nanosized $\mathrm{La}_{1-\mathrm{x}} \mathrm{Ca}_{\mathrm{x}} \mathrm{FeO}_{3}(0.00 \leq \mathrm{x} \leq 0.40)$ compounds prepared via sol-gel method followed by heat treatment at $1100^{\circ} \mathrm{C}$ for 24 hours are studied. Crystal structure, microstructure, surface morphology and temperature-dependent resistivity of the samples are investigated. TEM investigation reveals nanoparticles with an average size of $35 \mathrm{~nm}$ produced from the sol-gel process. The crystal structure of the compounds belongs to an orthorhombically distorted perovskite structure with Pbnm space group. Lattice distortion and cation size mismatch increase with an increase in $\mathrm{Ca}$ and particle and grain growth are suppressed by $\mathrm{Ca}$ doping. Electrical conduction is explained via thermally activated hopping of small polarons. Unit cell volume, charge ordering temperature, and activation energy for small polarons decrease linearly with an increase in cation size mismatch. Room temperature resistivity decreases with $\mathrm{Ca}$ doping and gets its minimum value for $30 \% \mathrm{Ca}$ at which the orthorhombic distortion is maximum.
\end{abstract}

Keywords-cation size mismatch; lattice distortion; particle size; grain size; charge order

\section{INTRODUCTION}

Rare-earth $(\mathrm{Re})$ transition metal perovskite oxides attract attention due to their electronic and magnetic properties, such as insulator to metal and paramagnetic to ferromagnetic phase transitions, magnetoresistance, electroresistance, magnetocaloric effect and charge order properties. $\mathrm{LaFeO}_{3}$ is a semiconductor/insulator perovskite oxide and its derived compounds by replacement of some of La atoms by other Re or alkaline earth (Ae) elements give rise to significant physical and chemical properties with promising applications in advanced technologies as in electronics, magnetics, fuel cells, gas sensors, and catalysts [1]. By partially exchanging La in the parent compound with other Re or $\mathrm{Ae}\left(\mathrm{La}_{1-\mathrm{x}} \mathrm{A}_{\mathrm{x}} \mathrm{FeO}_{3}, \mathrm{~A}\right.$ : Re or $\mathrm{Ae}$ ) lead to structurally distorted compounds, and induce different lattice parameters and unit cell volumes [2-4]. In addition, Ae doping has an influence on particle size (PS) and grain size $\left(\mathrm{R}_{\mathrm{G}}\right)$. The former is calculated using XRD data and the later is calculated using surface images taken by Scanning Electron Microscope (SEM) or Atomic Force Microscope (AFM). PS has an effect on electronic, magnetic and chemical properties and is an important parameter in determining the performance of the material.
Lack of holes or a trace of holes due to nonstoichiometric oxygen undoped compound has high resistivity at room temperature. Ae doping increases the number of holes in the compound and the polyvalent nature of the Fe compensates the created charge imbalance by oxidation. Ae with a valance state of + oxidizes two $\mathrm{Fe}^{+3}$ to two $\mathrm{Fe}^{+4}$ and Ae with a valance state of $2+$ oxidizes one $\mathrm{Fe}^{+3}$ to $\mathrm{Fe}^{+4}$. Therefore, the charge imbalance created by Ae doping is removed, and this process also increases holes in the compound leading to lower resistivity. Besides, oxygen content plays an important role in charge compensation. Oxygen deficiency leads to decrease the number of $\mathrm{Fe}^{4+}$, and excess oxygen leads to increase the number of $\mathrm{Fe}^{4+}$. Furthermore, doping at La site and $\mathrm{O}$ vacancy, have a direct impact on the local environment of Fe ions [4] and thereby resistivity. In addition, the difference of ionic radii of La-site ions in the compound and the difference of ionic radii of $\mathrm{Fe}^{+3}$ and $\mathrm{Fe}^{+4}$ have an influence on lattice and electrical properties. The measure of distortion due to La-site ionic radius difference is the cation size mismatch, $\sigma^{2}=\sum_{i} x_{i} r_{i}^{2}-\left\langle r_{A}\right\rangle^{2}$, where $x_{i}$ and $r_{i}$ are the concentration and ionic radius of the $i^{\text {th }}$ A-site ion and $\left\langle r_{A}\right\rangle$ is the average A-site ionic radius. The electrical transport in doped LFO compounds usually takes place by the "polaron hopping". An electron or a hole is trapped by a potential well resulting from the ionic displacements it created. Moreover, oxygen vacancy, which is a defect site, traps an extra electron and leads to polaron like bound states [5]. Charge Order (CO) is another property in some of these compounds which takes place in a material by a localization of charge carriers below a certain temperature, $\mathrm{T}_{\mathrm{CO}}$, giving rise to long-range order throughout the crystal structure leading a sudden increase in resistivity.

The electrical transport and the structural properties of $\mathrm{La}_{1}$ ${ }_{x} \mathrm{~A}_{\mathrm{x}} \mathrm{FeO}_{3}$ have been studied extensively. In $\mathrm{ReFeO}_{3}$ oxides having an orthorhombic perovskite structure with $\mathrm{Pbnm}$ space group, the structural distortion increases with decrease in $\mathrm{Re}^{3+}$ ionic radii [6]. It was shown that a decrease in ionic radii of $\mathrm{Re}^{3+}$ leads to decrease in lattice parameters and unit cell volume. Authors in [7] reported that doping the LFO with $\mathrm{Li}^{+}$ destroys the perfection of crystallinity and lead to decrease of PS. Authors in [8], contrary to other studies, reported that hightemperature synthesis of $\mathrm{K}^{+}$doped LFO doping with an ion having larger ionic radii than that of $\mathrm{La}$, leads to a decrease of 
unit cell volume. An increase in $\mathrm{Mg}^{2+}$ doping in LFO leads to lowered PS and doping with $\mathrm{Mg}^{2+}$ prevents PS from growing up during high temperature treatment [2]. However, despite the smaller ionic size of $\mathrm{Mg}^{2+}$ there was no considerable change in unit cell volume up to $70 \%$ doping. A study on $\mathrm{Ba}^{2+}$ doped LFO revealed that an increase in doping decreased the PS and contrary to the larger ionic size of $\mathrm{Ba}^{2+}$ the unit cell volume decreased and appropriate Ba-doping restrained the growth of grains [9]. Moreover, the effect of $\mathrm{Pb}^{2+}$ doping on the structural properties of LFO was conducted in [10] with no change in the unit cell volume and a decrease in the PS with an increase in $\mathrm{Pb}^{2+}$ content [10]. An increase in $\mathrm{Ca}^{2+}$ in LFO leads to decrease in PS $[11,12]$ and in grain size [13]. Structural and electrical properties of simultaneously doping LFO with $\mathrm{Ca}^{2+}$ and $\mathrm{Pb}^{2+}$ [14] showed that doping with larger $\mathrm{Pb}^{2+}$ ions up to $20 \%$ did not change the principal phase, but lattice parameters, unit cell volume and PS increased. However, another study reported that $\mathrm{Pb}^{2+}$ doping lead PS to decrease [15]. Electrical transport properties of LFO can be tuned by alkaline ion substitution. The incorporation of $\mathrm{Ca}^{2+}$ ions into the La sub-lattice decreased the lanthanum ferrite's electrical resistivity $[16,17]$. Moreover, doping the parent compound LFO with another divalent ion, $\mathrm{Mg}^{2+}$, led to increase the resistance of LFO due to secondary phases and nonstoichiometric oxygen in the structure [2].

Although there are studies involving the effect of divalent ion doping on the structural properties of the $\mathrm{LaFeO}_{3}$ compound, a detailed and combined study on the effect of divalent ion doping on structural parameters, microstructure, and electrical properties is required. Therefore, in this study, the effect of cation size mismatch on the unit cell volume, charge order temperature, compound activation energy, microstructure, particle size, and grain size evaluation are discussed in detail. Using divalent $\mathrm{Ca}^{2+}$ as a dopant ion, crystal structure, microstructure, and the electrical properties of $\mathrm{La}_{\mathrm{x}} \mathrm{Ca}_{1-\mathrm{x}} \mathrm{FeO}_{3}(\mathrm{x}=0.10,0.20,0.30,0.40$, ) perovskites prepared by standard sol-gel route are considered.

\section{EXPERIMENATAL PROCEDURE}

$\mathrm{La}_{1-\mathrm{x}} \mathrm{Ca}_{\mathrm{x}} \mathrm{FeO}_{3}(0.0 \leq \mathrm{x} \leq 0.4)$ samples have been prepared by standard sol-gel method, using appropriate amounts of $\mathrm{La}\left(\mathrm{NO}_{3}\right)_{3} \cdot 6 \mathrm{H}_{2} \mathrm{O}, \mathrm{Ca}\left(\mathrm{NO}_{3}\right)_{2} \cdot 4 \mathrm{H}_{2} \mathrm{O}, \quad \mathrm{Fe}\left(\mathrm{NO}_{3}\right)_{3} \cdot 9 \mathrm{H}_{2} \mathrm{O}$ with the desired stoichiometry. Pellets pressed using the fine powders were sintered at $1100^{\circ} \mathrm{C}$ for $24 \mathrm{~h}$ and cooled down to room temperature in the furnace. Close cycle cryostat was used to perform temperature dependent resistivity measurement using four-point probe from $320 \mathrm{~K}$ down to $140 \mathrm{~K}$. Details of the experimental procedure can be found in [18].

\section{RESULTS AND DISCUSSION}

\section{A. Crystal Structure Analysis}

The room temperature structures of the $\mathrm{La}_{1-\mathrm{x}} \mathrm{Ca}_{\mathrm{x}} \mathrm{FeO}_{3}$ $(0.0 \leq x \leq 0.4)$ samples were investigated using their respective $\mathrm{X}$-ray diffraction profiles. To explore the effect of $\mathrm{Ca}$ doping in detail, the structural parameters of all the samples were refined by Rietveld's profile-fitting using freely available FullProf Suit software [19]. The given structural model was refined with the peak shapes of the experimental patterns were assumed to be a Thompson-Cox-Hastings (TCH) pseudo-Voigt profile functions. Lattice parameters, particle size and lattice strain were refined to fit the peak shifting and broadening of the experimental data. The values of goodness of fit $(\mathrm{GoF})$ for all of the refinement lie between 0.87 and 1.3. GoF was calculated by the ratio $R_{e} / R_{w p}$, where $R_{e}$ and $R_{w p}$ are the expected weighted and weighted profile factors. The experimental and calculated profiles with their differences were figured out along with Bragg positions (see Figure 1). Rietveld refinement reveals that the samples crystallize in orthorhombically distorted perovskite structure with $\mathrm{Pbnm}$ space group. Inspecting on main peak, in Figure 1 (XRD pattern of LCFO40) and in Figure 2, there emerges another peak (marked with $\mathrm{a} *$ ) in the pattern of LCFO40.
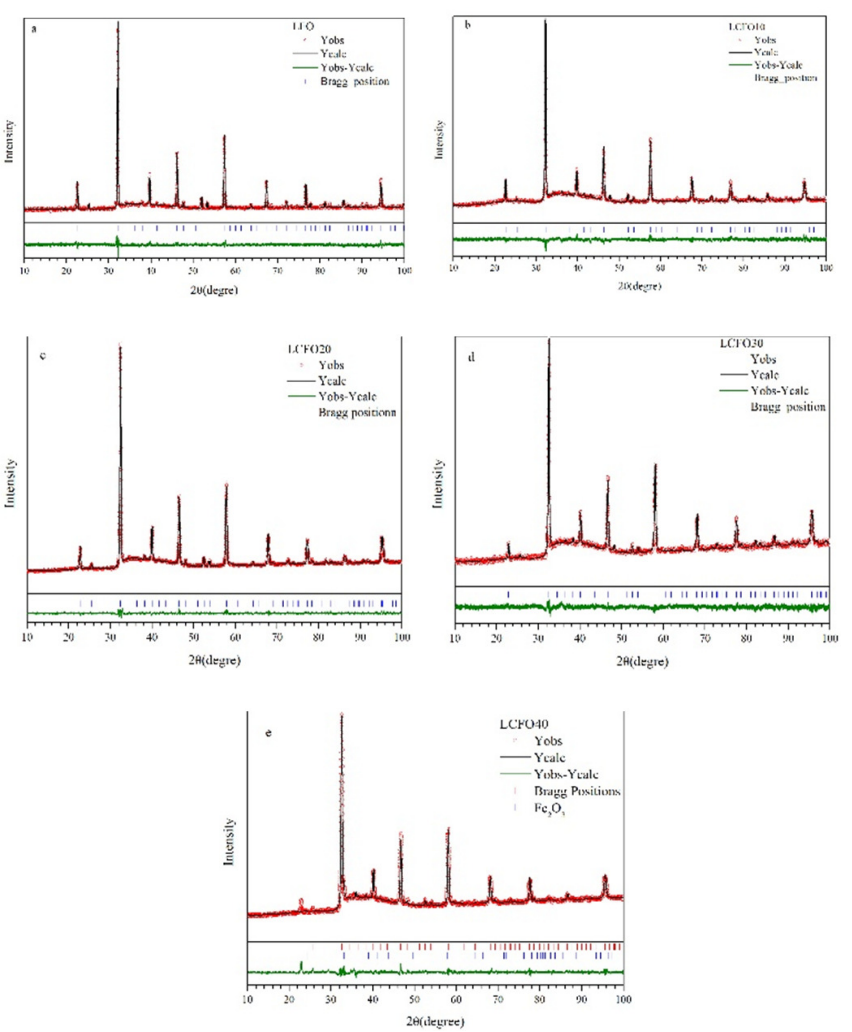

Fig. 1. The experimental and calculated XRD patterns of all samples. Open circles indicate the experimental data and the calculated data are shown in the continuous line overlapping them. The curve (at the bottom) shows the difference between the experimental and calculated patterns. The vertical bars indicate the expected reflection positions for the main (blue-upperbars) and $\mathrm{Fe}_{2} \mathrm{O}_{3}$ impurity (red-lower bars) phases.

Further investigation revealed that this peak was due to the trace of the rhombohedrally distorted perovskite $\mathrm{Fe}_{2} \mathrm{O}_{3}$ (hematite) with $R \overline{3} c$ space group (Crystallographic Open Data (COD) base card \# 96-901-0140). Refined lattice parameters, unit cell volumes, average A-site radii, $\left\langle r_{A}\right\rangle$, average distortion ratio (D), cation size disorder $\left(\sigma^{2}\right)$, and Rietveld refinement parameters are summarized in Table I. Inspection on the XRD patterns of the samples makes clear that following $\mathrm{Ca}$ doping, main-peak of parent compound LFO at $32.17^{\circ}$ (Figure 2) is rendered broader and shifted to higher angles. It is known that the former is due to decrease in particle size and the latter is a change of the lattice constants. 


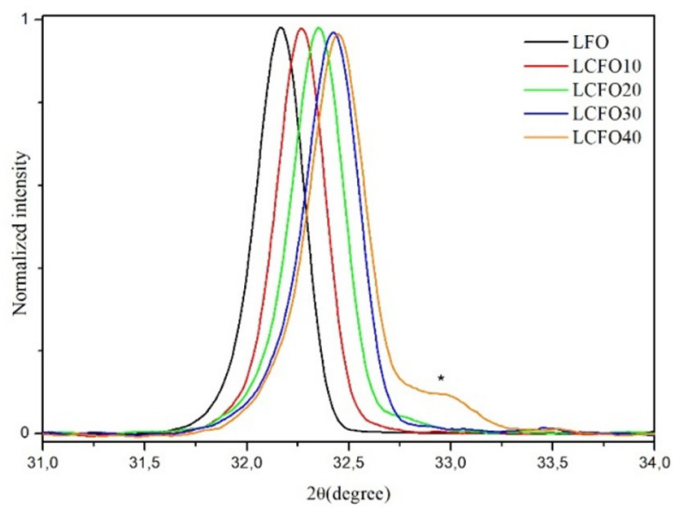

Fig. 2. Main peaks of LCF, LCFO10, LCFO20, LCFO30, and LCFO40 samples with * indicating the main peak of the $\mathrm{Fe}_{2} \mathrm{O}_{3}$ phase

Calculated unit cell volumes for all samples after Reitveld refinement are given in Table I. Unit cell volume calculated for the parent compound LFO is $242.72 \AA^{3}$. A $10 \%$ increase in $\mathrm{Ca}$, results to smaller unit cell with volume $239.85 \AA^{3}$. Further increase in $\mathrm{Ca}$ leads to decreased unit cell volumes: $238,04 \AA^{3}$, $235.26 \AA^{3}$, and $235.12 \AA^{3}$ for $20 \%, 30 \%$, and $40 \%$ Ca respectively. Therefore, an increase in $\mathrm{Ca}$ amount decreases the unit cell volume which agrees with the replacement of $\mathrm{La}^{3+}$ having 12 coordinated ionic radii of $1.36 \AA$ with the smaller $\mathrm{Ca}^{+2}$ ion with 12 coordinated ionic radii of $1.34 \AA$. Doping the parent compound with smaller ion decreased $\left\langle r_{A}\right\rangle$ thus unit cell volume decreases. Moreover, there is another contribution on shrinkage of the unit cell which can be explained by parent compound doping, $\mathrm{LFO}$, with $\mathrm{Ca}^{2+}$ ion resulting to oxidation of $\mathrm{Fe}^{3+}$ to $\mathrm{Fe}^{4+}$. An increase in doping leads to a decrease in concentration of $\mathrm{Fe}^{3+}(0.645 \AA)$ with larger ionic radii, and an increase of concentration of $\mathrm{Fe}^{4+}(0.585 \AA)$ with smaller ionic radii. Hereby, there is a decrease in size around Fe-site which may also contribute to a decrease of unit cell volume [20].

TABLE I. LATTICE PARAMETERS

\begin{tabular}{|c|c|c|c|c|c|c|c|c|c|c|c|}
\hline $\mathbf{x}(\%)$ & $a(\AA ̊)$ & $b(\AA ̊)$ & $c(\AA ̊)$ & $V\left(\AA^{3}\right)$ & $\left\langle r_{A}\right\rangle(\AA)$ & $D(\%)$ & $\sigma^{2}\left(10^{-4} \AA^{2}\right)$ & $R_{p}(\%)$ & $R_{w p}(\%)$ & $\chi^{2}$ & GoF \\
\hline 0.00 & 5.5598 & 5.5565 & 7.8569 & 242.72 & 1.360 & 0.06 & 0.000 & 24.2 & 13.4 & 1.66 & 1.3 \\
\hline 0.10 & 5.5399 & 5.5353 & 7.8216 & 239.85 & 1.358 & 0.11 & 0.360 & 34.5 & 18.4 & 1.45 & 1.2 \\
\hline 0.20 & 5.5075 & 5.5162 & 7.8024 & 238.04 & 1.356 & 0.15 & 0.640 & 22.4 & 13.5 & 1.13 & 1.1 \\
\hline 0.40 & 5.5045 & 5.4935 & 7.7755 & 235.12 & 1.352 & 0.14 & 0.960 & 23.9 & 14.1 & 0.75 & 0.87 \\
\hline
\end{tabular}

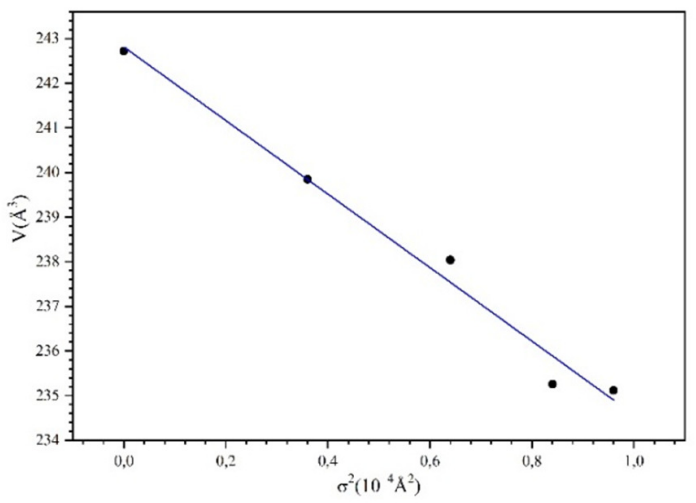

Fig. 3. Change of unit cell volume with respect to cation size mismatch. The line represents the fitting result.

Even expressing the decrease in unit cell volume with $\mathrm{Ca}$ doping, it will be better to express the change in the unit cell volume with respect to A-site cation size mismatch $\left(\sigma^{2}\right)$ which is an inherent parameter for doped perovskite structures and it measures the size disorder of the structure [21]. To represent the change of unit cell volume, $\mathrm{V}$ is plotted with respect to $\sigma^{2}$ which is given in Figure 3. It is clear from the Figure that as the cation size mismatch increases the unit cell volume decreases linearly. Therefore, fitting the data with following linear equation in $\sigma^{2}$ was performed:

$$
V\left(\sigma^{2}\right)=V_{0}-m \sigma^{2}
$$

where $V_{0}$ represents the volume of the sample at which $\sigma^{2}$ is zero (namely $V_{0}$ is the volume of the parent compound, LFO) and $m$ is the slope of the line. Fitting results to $\chi^{2}=0.98, V_{0}$ and $m, 242.81 \pm 0.41 \AA^{3}$ and $-82373 \pm 6268 \AA$ respectively. The calculated unit cell volume for the parent compound using linear fitting is almost equal to the value $\left(242.72 \AA^{3}\right)$ from Rietveld refinement. It is seen from Table I and Figure 3 that there is no considerable change in the volume of LCFO40 as it is expected due to $10 \%$ more Ca with respect to LCFO30. For LCFO40, the amount of $\mathrm{Ca}$ in the structure may be lower than the expected. This can be explained with the appearance of $\mathrm{Fe}_{2} \mathrm{O}_{3}$ as an impurity phase in LCFO40 (Figure 1). If some $\mathrm{Fe}$ atoms are not in the main phase, then there should be the compounds or the oxides of $\mathrm{Ca}$ and/or La. However, there is no trace of the compound or the oxide of $\mathrm{La}$ and/or $\mathrm{Ca}$ in the XRD pattern. An amount of impurity phase which should be dispersed in the main perovskite phase and the precipitates with low concentration usually exhibit particle sizes in the range of a few nm, invisible to the XRD [22].

Doping an orthorhombic perovskite component at the Asite with an element having different ionic radii results to deviation from the cubic symmetry. The measure of deviation is an average distortion ratio, $D$, which is defined as [23]:

$$
D=\frac{1}{3} \sum_{i=1}^{3}\left[\frac{\left(a_{i}-\langle a\rangle\right)}{a_{i}}\right]
$$

where $\langle a\rangle=(a b c / \sqrt{2})^{1 / 3}, a_{1}=a, a_{2}=b, a_{3}=c / \sqrt{2}$, and $a$, $b$, and $c$ are calculated lattice parameters. The calculated values of $D$ for all of the compounds are given in Table I. The average distortion ratio $D$, exhibits a drastic increase from $0.6 \%$ to $0.11 \%$ for $10 \%$ and $20 \%$ Ca respectively which implies a larger orthorhombic distortion from sample LCFO10 to LCFO20 and it increases monotonically with increase in $\mathrm{Ca}$. However, there is a distortion decrease for the sample doped with $40 \% \mathrm{Ca}$ compared to the $30 \%$ sample. One can argue that this is compatible with $\mathrm{Fe}_{2} \mathrm{O}_{3}$ as an impurity in the structure, which 
may indicate that the $\mathrm{Ca}$ amount in the compound is less than $40 \%$.

\section{B. Microstructural Analysis: Particle Size and Strain}

Particle size and lattice strain $(s)$ are parameters which can be extracted from the peak width analysis. Particle size generally has been calculated using Scherrer equation and Williamson-Hall (W-H) method. The full width at half maximum of the most intense peak for a given pattern is used in Scherrer's equation $(D=K \lambda / \beta \cos \theta)$. Without correction for the lattice strain, this gives a rough particle size. One can say that, Scherrer's equation gives only an approximate estimate of the mean particle size. But, the peak broadening due to size and strain is expressed in Williamson-Hall method as:

$$
\beta_{h k l} \cos \theta=\frac{K \lambda}{D}+4 \epsilon \sin \theta
$$

where $\beta_{h k l}$ is the full with at half maximum of the corresponding reflections in XRD pattern, $K$ is the shape factor, $\lambda$ is the X-Ray wavelength, $D$ is particle size and $\varepsilon$ is strain. Plotting $\beta_{h k l} \cos \theta$ values with respect to $4 \sin \theta$ is called a Williamson-Hall plot. Fitting the data with a linear equation gives the strain and particle size from the slope and y-intercept of the fitted line respectively [24]. The FullProf Suite program was used to calculate the microstructural parameters of the sample. The microstructural effects were handled using the Voigt approximation: both instrumental and sample intrinsic profile are supposed to be described approximately by a convolution of Lorentzian and Gaussian components [19].

TABLE II. AVERAGE PARTICLE SIZE, AND AVERAGE GRAIN SIZE

\begin{tabular}{|c|c|c|c|}
\hline Ca (\%) & $\langle\boldsymbol{P S}\rangle(\mathbf{n m})$ & $\boldsymbol{S}$ & $\boldsymbol{R}_{\boldsymbol{G}}(\mathbf{n m})$ \\
\hline 0 & $77.18 \pm 0.08$ & $1.95 \pm 0.02$ & 940 \\
\hline 10 & $56.43 \pm 0.01$ & $18.25 \pm 0.03$ & 670 \\
\hline 20 & $51.43 \pm 0.05$ & $25.17 \pm 0.01$ & 620 \\
\hline 30 & $33.12 \pm 0.05$ & $17.39 \pm 0.00$ & 450 \\
\hline 40 & $24.06 \pm 0.02$ & $6.48 \pm 0.01$ & 540 \\
\hline
\end{tabular}

The parent compound LFO has the largest particles with average size of $77.18 \pm 0.08 \mathrm{~nm}$. The doping parent compound with $10 \% \mathrm{Ca}$, had an average PS decrease to $56.43 \pm 0.01 \mathrm{~nm}$. PS gets its minimum value for LCFO40 at $24.06 \pm 0.02 \mathrm{~nm}$. As doping increases, the average PS decreases. The average strain for the parent compound is 1.95 which is the smallest, while $10 \% \mathrm{Ca}$ doping increased strain to 18.25 . Further increase in Ca with $10 \%$ leads strain to attain its maximum for LCFO20. Doping more $\mathrm{Ca}$ decreases strain. The average strain is calculated as 6.48 for the LCFO40 sample with $40 \% \mathrm{Ca}$. The calculated particle size and strain for the samples are given in Table II, and the plot representing the change in these parameters with respect to $\mathrm{Ca}$ is given in Figure 4. It is clear from the plot that as Ca doping increases, PS size decreases. The particle size of the doped oxide becomes smaller than the pure host oxide, as a consequence of the restriction of the motion of particles at the boundaries between host and dopant particles [25]. The results obtained in this study are consistent with findings in $[2,8-12,26]$, even if these studies have concluded to a decrease in average PS with an increase in doping. The method used in these studies to calculate average PS is only taking the contribution of the main peak in the XRD pattern. It is well established that, as the particle size decreases, surface and interface between the particles increase. And, surfaces are the place of broken bonds and oxygen vacancies which increase stress and strain on the surface and interface of the particles [27].

\section{Atomic Force Microscopy Analyss}

Surface scanning of the samples was performed by AFM in non-contact mode with a resolution of $5 \mu \mathrm{m} \times 5 \mu \mathrm{m}$. Scanned images of all samples are given in Figure 5. These images reveal that larger grains are surrounded by smaller ones and they are tightly connected with each other. This introduces that the grain growth process is not finalized yet. The average grain size calculated for the undoped sample is $940 \mathrm{~nm}$. Grain size decreases from $940 \mathrm{~nm}$, as nominal Ca increases in the samples up to $30 \% \mathrm{Ca}$ for which grain size is $450 \mathrm{~nm}$. The grain size of the doped sample becomes smaller than the pure host sample $[9,13,28]$, and the sample with $40 \% \mathrm{Ca}$ has an average grain size of $540 \mathrm{~nm}$. A decreasing trend in grain size up to $40 \% \mathrm{Ca}$ is consistent with the decreasing trend of particle size with $\mathrm{Ca}$ content. However, the average grain size of the sample LCFO40 was calculated to be $540 \mathrm{~nm}$. This increase in grain size (from $450 \mathrm{~nm}$ to $540 \mathrm{~nm}$ ) may result from a Ca deficiency in the sample LCFO40 and impurity phase $\mathrm{La}_{2} \mathrm{FeO}_{3}$. The change in $\mathrm{R}_{\mathrm{G}}$ with $\mathrm{Ca}$ content is given in Figure 4. The largest particles calculated for the parent compound are $77.18 \pm 0.08 \mathrm{~nm}$. The sample with the largest average PS has the largest average grain size $(940 \mathrm{~nm})$. Average PS is smaller than the average grain size calculated from AFM images (Table II).

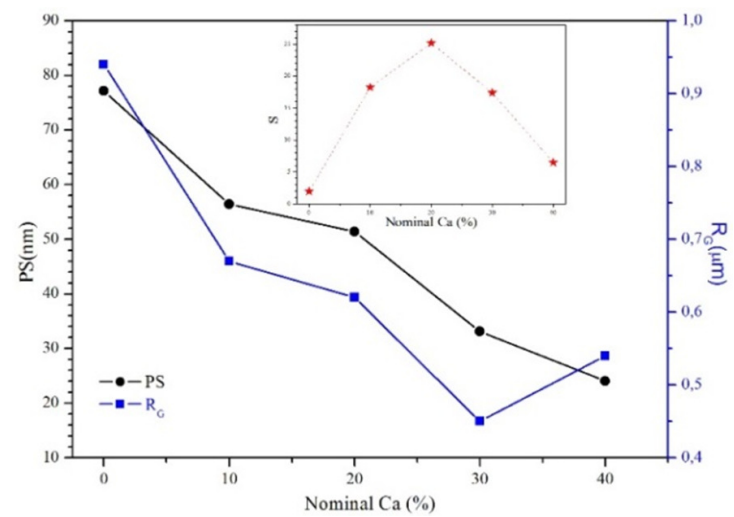

Fig. 4. Variation of particle and grain size as a function of nominal Ca. Inset represents the change of strain with nominal $\mathrm{Ca}$.

This suggests that, the grains viewed by AFM images are agglomerates of almost a few thousands $\left(\frac{R_{G}}{P S}\right)^{3}$ of particles [29]. Grain size calculated using the AFM image is a local average over tens of grains, but particle size calculated using XRD data is an average namely over $10^{10}$ particles. One can say that particle size calculated using XRD pattern with $\mathrm{W}-\mathrm{H}$ analysis is a more accurate method. The change of microstructural strain with respect to $\mathrm{Ca}$ is given in Figure 4 as an inset. Since the sign of the strain is positive, it stands for compressive strain. Generally, there is a trend of increasing and decreasing strain with $\mathrm{Ca}$. The samples undergo increasing compressive strain up to $20 \% \mathrm{Ca}$ and then decrease up to $40 \% \mathrm{Ca}$. 

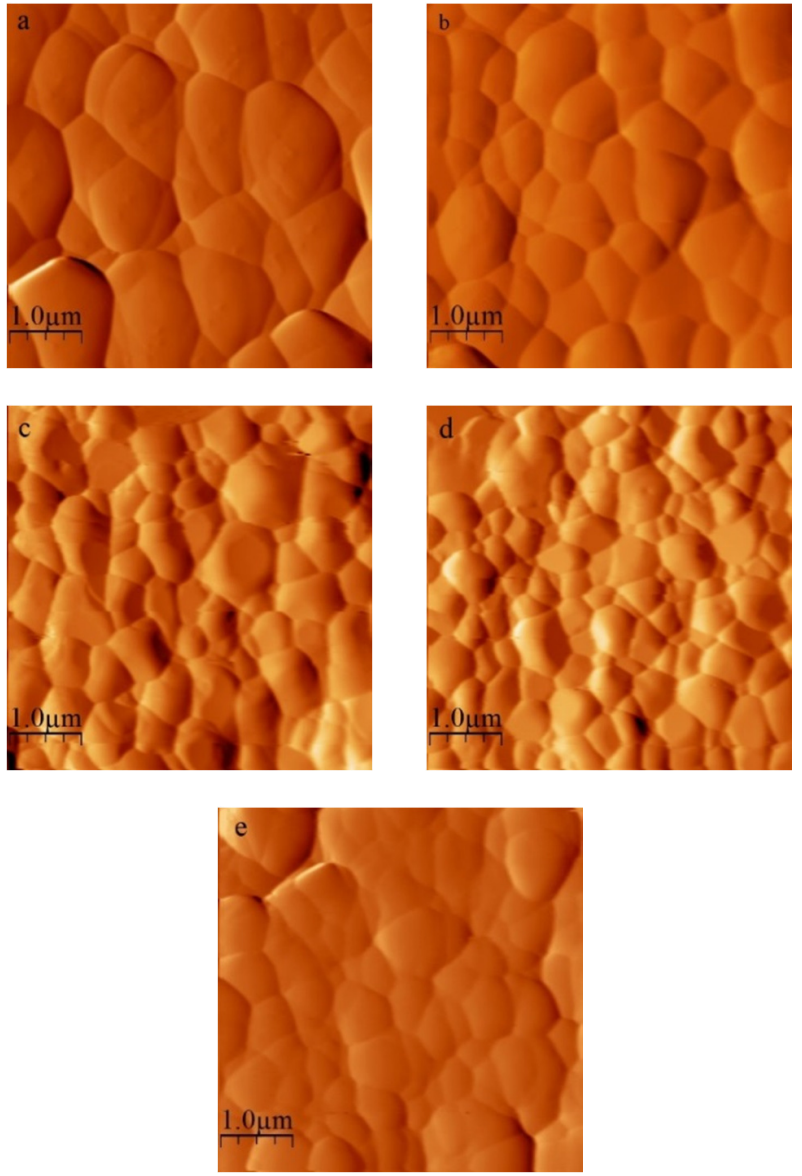

Fig. 5. $5 \mu \mathrm{m} \times 5 \mu \mathrm{m}$ AFM images of the samples, (a) LFO, (b) LCFO10, (c) LCFO20, (d) LCFO30, (e) LCFO40

\section{High-resolution TEM Analysis}

Surface morphology, particle size distribution and structure of the nanoparticles were studied by High-Resolution Transmission Electron Microscopy (HRTEM) using a JEOLJEM 2010 operated at $200 \mathrm{kV}$. Powder specimens were dispersed into acetone and underwent an ultrasonic bath for 20 minutes. A drop of solution was taken on a carbon grid to carry out HRTEM analysis. TEM investigation was performed to clarify the nanosized particle distribution and perfect crystallinity of LCFO20 precursor after calcination of the solgel route for $12 \mathrm{hs}$ at $600^{\circ} \mathrm{C}$. A general view of the particle distribution of LCFO20 precursor is given in Figure 6(a). It is clear from the image that nanoparticle distribution with size between $20 \mathrm{~nm}$ and $50 \mathrm{~nm}$ was produced from the sol-gel process. Though TEM images are not necessarily representative of the whole sample, the average value of particle size calculated from XRD analysis (Table II) was closely represented by them. For further structural investigation one of the nanoparticle's surface was focused (Figure 6(b)) and HRTEM technique was applied. Inverse Fast Fourier Transform (IFFT) of the real image indicated by a square in Figure 6(b) was taken in order to filter the unwanted noise (Figure 6(c)). The perfect crystalline structure of LCFO20 precursor before heat treatment is clear.
FFT diffraction pattern extracted by applying a rectangular Region of Interest (R-ROI) is given in the upper left inset of Figure 7(a). IFFT of the R-ROI of real image is given in the upper right inset of Figure 7(a). Five spots were identified which correspond to the distance between different lattice planes as $0.5929,0.4122,0.3124$, and $0.2048 \mathrm{~nm}$. These planes, having plane distances of $0.2601,0.3124$ and $0.2048 \mathrm{~nm}$, labeled as 1, 2 and 3 respectively, were indexed using lattice parameters of LCFO20 calculated from XRD data. The planes were indexed as follows: plane 1 was indexed as $\left(\begin{array}{lll}0 & 2 & 0\end{array}\right),\left(\begin{array}{ll}1 & 1\end{array}\right.$ 2), ( $\left.\begin{array}{lll}2 & 0 & 0\end{array}\right)$, plane 2 was indexed as $\left(\begin{array}{lll}1 & 1 & 1\end{array}\right)$ and plane 3 was indexed as $\left(\begin{array}{lll}0 & 0 & 4\end{array}\right)$ and $\left(\begin{array}{lll}2 & 2 & 0\end{array}\right)$. Theoretical calculation using lattice parameters of the sample LCFO20 gives an angle of $5.86^{\circ}$ between the planes $\left(\begin{array}{lll}2 & 2 & 0\end{array}\right)$ and $\left(\begin{array}{lll}1 & 1 & 1\end{array}\right)$, and $50.89^{\circ}$ between the planes $\left(\begin{array}{lll}2 & 0 & 0\end{array}\right)$ and $\left(\begin{array}{lll}1 & 1 & 1\end{array}\right)$. The two planes shown in Figure 7(a) form an angle of $50.06^{\circ}$ which was calculated by IFFT analysis. This result suggests that high-quality crystalline structure was obtained in this study. The line profile is given in Figure $7(\mathrm{~b})$ to emphasize interatomic distances. The average distance between the atoms was found to be $5.1 \AA$ which closely represents the lattice parameters $a$ and/or $b$ of the LCFO20 (Table I).

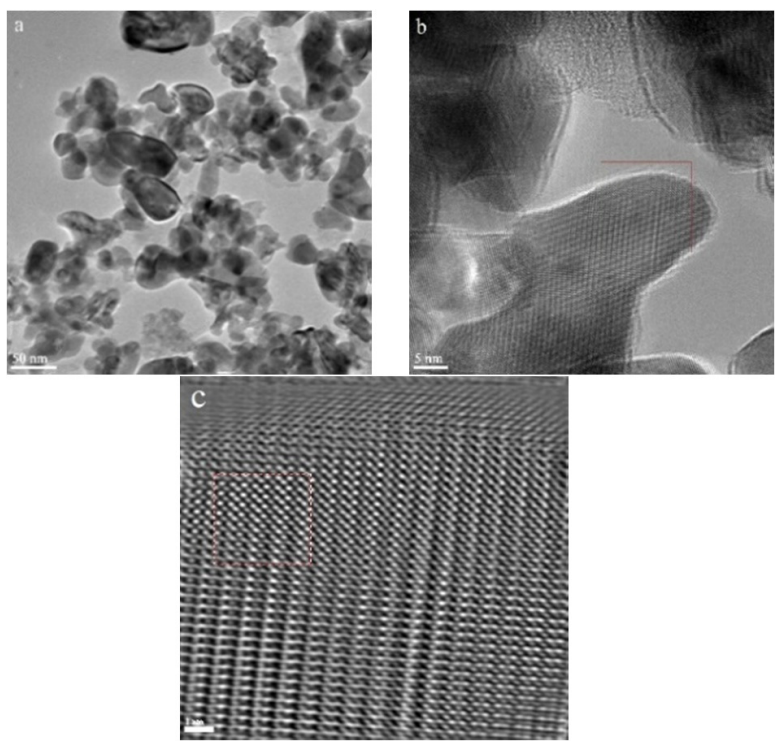

Fig. 6. (a) TEM image of the prepared LCFO20 taken after burning the dried jel at $600^{\circ} \mathrm{C}$, (b) HRTEM image of the particles with size 20nm, (c) IFFFT of the image in (b).
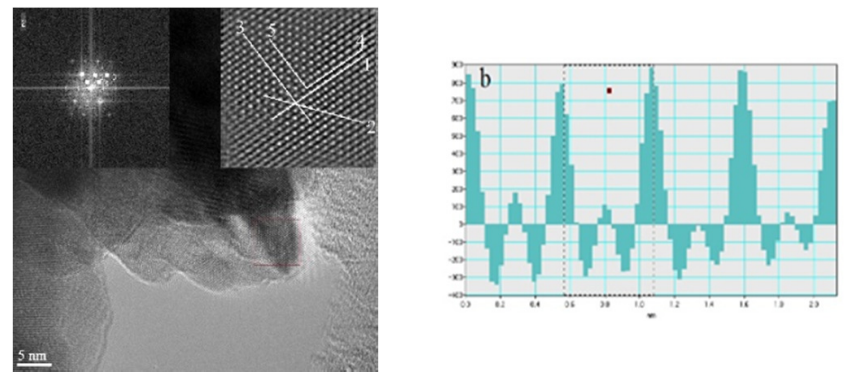

Fig. 7. High resolution TEM image (HRTEM) of LCFO20 after heath treatment at $1100{ }^{\circ} \mathrm{C}$. (a) FFT diffraction pattern extracted by applying rectangular R-ROI left inset, IFFT of the R-ROI of real image is given in the upper right inset. (b) The intensity profile of the atomic layers. 


\section{E. Electrical Properties}

Temperature dependence of the samples' resistance is given in Figure 8. The change of the resistance of the samples shows the same trend with temperature which exhibits insulating properties for the studied temperature range (140-320K). Moreover, all samples exhibit strong insulating behavior at low temperatures. Due to the compliance limit of the measuring device, the resistance of the parent compound LFO could not be measured. At high temperature, the resistance of the sample changes slightly and increases as the temperature decreases. However, at low temperatures, resistance increases abruptly. This abrupt increase in resistivity may indicate that the samples are charge ordered (CO) insulators. This phenomenon is another property of mixed valence perovskite oxide. The onset of $\mathrm{CO}$ phase can be triggered by temperature and causes a sudden increase in the transport properties of the perovskite and lattice degrees of freedom [30]. The charge order temperature, $T_{C O}$, is a temperature at which the onset of $\mathrm{CO}$ is observed. $T_{C O}$ can be calculated by taking the derivative of $\ln (\rho / T)$ against the $1 / T$ curve $(\mathrm{d}(\ln \rho) / \mathrm{d}(1 / T))$ and plotting the derivative as a function of temperature (Figure 9). The temperature at which a sharp peak within the derivative plot observed is the $T_{C O}$. The calculated values of $T_{C O}$ for all the samples are shown in Table III and the plot of the variation of $T_{C O}$ with $\sigma^{2}$ is given in Figure 9. For the samples LCFO10, LCFO20, LCFO30, and LCFO40 the calculated values are $197 \mathrm{~K}, 177 \mathrm{~K}, 161 \mathrm{~K}$, and $164 \mathrm{~K}$. There is a decreasing trend in $T_{C O}$ with increase in $\mathrm{Ca}$ except for LCFO40. Ca deficiency and $\mathrm{Fe}_{2} \mathrm{O}_{3}$ as an impurity phase in LCFO40 may lead to an increase of $T_{C O}$ for this sample.

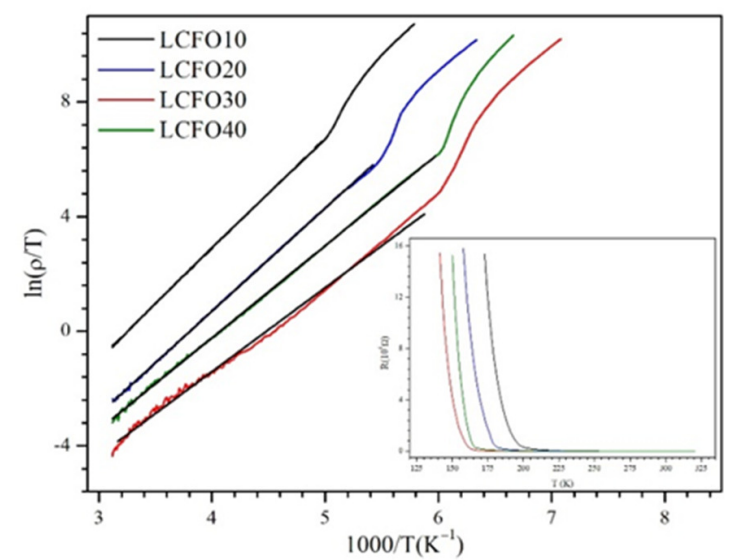

Fig. 8. Plot of $\ln (\rho / T)$ vs $1 / T$. The continuous lines represent the linear fitting. The temperature dependent resistances of the samples are given in the inset.

TABLE III. DEBYE TEMPERATURE, ACTIVATION ENERGY AND $\rho_{300 K}$

\begin{tabular}{|c|c|c|c|c|}
\hline $\mathbf{C a}(\boldsymbol{\%})$ & $\boldsymbol{\theta}_{\boldsymbol{D}}(\mathbf{K})$ & $\boldsymbol{E}_{\boldsymbol{a}}(\mathbf{m e V})$ & $\boldsymbol{\rho}_{\boldsymbol{0}}(\boldsymbol{\mu} \mathbf{\Omega c m})$ & $\boldsymbol{\rho}_{\mathbf{3 0 0 \boldsymbol { K }}}(\boldsymbol{\Omega c m})$ \\
\hline 10 & 400 & $332.57 \pm 0.36$ & $3.59 \pm 0.06$ & 405.16 \\
\hline 20 & 368 & $312.45 \pm 0.41$ & $1.14 \pm 0.02$ & 52.12 \\
\hline 30 & 332 & $287.65 \pm 0.54$ & $0.49 \pm 0.04$ & 10.47 \\
\hline 40 & 320 & $276.57 \pm 0.46$ & $2.11 \pm 0.05$ & 29.37 \\
\hline
\end{tabular}

The resistivity of all samples increased rapidly after $\mathrm{CO}$ took place, due to the localization of electronic states which characterize the $\mathrm{CO}$ state. Changing the number of charge carriers by hole doping $\left(\mathrm{Ca}^{2+}\right.$ amount in the sample) has an effect on $T_{C O}$, but hole doping alone cannot describe the change of CO temperature. The structural factors including structural distortion or cation size disorder influence the electrical transport properties of these compounds. Electrical properties can be modified by changing the type and the concentration of trivalent $\mathrm{Re}$, divalent or monovalent Ae cations, which led distorted crystal structure due to ionic size differences [31]. In our study, $\mathrm{La}^{3+}$ and $\mathrm{Ca}^{2+}$ have different ionic radii coordinated by 12 nearest $\mathrm{O}$ ions in the perovskite structure $\left(\left\langle\mathrm{r}_{\mathrm{La}}\right\rangle=1.36 \AA\right.$ and $\left\langle\mathrm{r}_{\mathrm{Ca}}\right\rangle=1.34 \AA$ ).

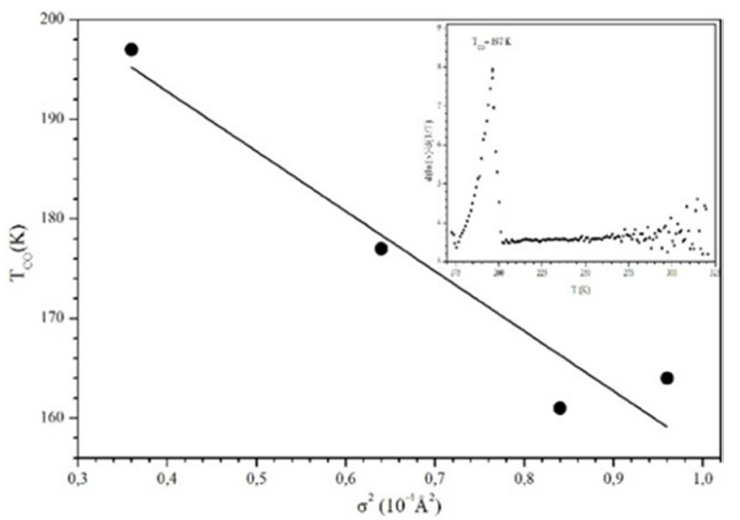

Fig. 9. Plot of $T_{C O}$ vs $\sigma^{2}$. The line represents the fitting result. Inset, the plot of the derivative of $\ln (\rho / T)$ curve with respect to $T$.

The concentration of the cations and their ionic radii differences determine the distortions of crystal structure [31, 32]. The plot of $T_{C O}$ with $\sigma^{2}$ is used to express the influence of a measure of this distortion at the A-site on $T_{C O}$ (Figure 9). A linear decrease of $T_{C O}$ with $\sigma^{2}$ is clear. Linear variation of $T_{C O}$ versus $\sigma^{2}$ was analyzed with [33]:

$$
T_{C O}\left(\sigma^{2}\right)=T_{C O}(0)-m \sigma^{2}
$$

where $T_{C O}(0)$ represents the estimated value of the $T_{C O}$ of a parent compound in the given series and $m$ is the proportionality factor which was obtained from the slope of the corresponding figure. Fitting gives $T_{C O}(0)=224.20 \pm 1.53 \mathrm{~K}$, and slope $m=-18.69 \times 10^{-4} \mathrm{~K} / \AA^{2}$ with reduced chi-square of 0.97 . Due to the compliance of the measurement devices $\rho(T)$ for the parent compound, LFO was not performed. But, for the parent compound, $T_{C O}$ was estimated to be $224.20 \mathrm{~K}$ with the help of (4) and fitting results. $224.20 \mathrm{~K}$ is the estimated highest possible CO temperature of the parent compound with no mismatch, which is consistent with the results of [34]. With a linear decrease in $T_{C O}$ with $\sigma^{2}$ we may conclude that upon increasing A-site disorder, i.e. an increase in $\sigma^{2}$ from 0.0 to $1.0 \times 10^{-4} \AA^{2}$, the $\mathrm{CO}$ is gradually suppressed. Since ionic radii of $\mathrm{Ca}^{2+}$ are close to ionic radii of $\mathrm{La}^{3+}\left(\left\langle\mathrm{r}_{\mathrm{La}}\right\rangle=1.36 \AA\right.$ and $\left\langle\mathrm{r}_{\mathrm{Ca}}\right\rangle=1.34 \AA$, the change of $\sigma^{2}$ even at high doping with $\mathrm{Ca}^{2+}$ will be small. However, using dopant elements with larger ionic size may give high disorder at A-site, which may lead to collapse of charge order for a given composition. Typical plots of temperature variations of electrical resistivity for all samples are shown in Figure 8. The resistivity axis is represented with 
$\ln (\rho / T)$ and temperature axes with $1 / T$ scale. All samples exhibit insulating behavior. Thermally activated hopping of small polarons is a model which describes the resistivity of perovskite oxides $[35,36]$. Therefore, the resistivity was analyzed with the use of the Small Polaron Hopping (SPH) model [37]. According to the SPH model, the expression for the temperature dependence of the electrical resistivity is given by [38]:

$$
\rho=\rho_{o} T \exp \left(\frac{E_{a}}{k_{B} T}\right)
$$

where $\rho_{0}$ is a pre-exponential factor, $T$ is the absolute temperature, $k_{B}$ is the Boltzmann constant and $E_{a}$ represents the activation energy for small polarons. Calculated Debye temperatures are given in Table III (the temperature at which resistivity deviates from linearity is $\left.\theta_{D} / 2\right)$. In the high temperature region, linear increase of $\ln (\rho / T)$ with $1 / T$ is clear for all samples (Figure 8). Therefore, resistivity data in the given temperature range, (temperatures higher than $\theta_{D} / 2$ ) fit to a linear equation. From the fitting results of the best fitted lines, the slope gives $E_{a} / k_{\mathrm{B}}$ and the intercept gives $\rho_{0}$. The results of $E_{a}$ for all samples are given in Table III. An increase in Ca leads to a decrease in $E_{a}$ from $332.57 \pm 0.36 \mathrm{meV}$, to $312.45 \pm 0.41 \mathrm{meV}$ for samples LCFO10 and LCFO20 respectively. The calculated activation energies for samples LCFO30 and LCFO40 are $287.65 \pm 0.54 \mathrm{meV}$ and $276.57 \pm 0.46 \mathrm{meV}$. As the doping increases, $E_{a}$ decreases for all the samples, in accordance to $[2,16,17]$. The perovskite oxides are classified in terms of their conduction mechanism based on the values of $E_{a}$. If $E_{a}>1 \mathrm{eV}$ then that material is oxide ion conductor $[39,40]$. Whereas, it is p-type polaronic insulator if $900 \mathrm{meV}>E_{a}>200 \mathrm{meV}$ and it is an n-type polaronic insulator if $E_{a} \leq 200 \mathrm{meV}$ [41, 42]. Therefore, the activation energies calculated for samples in this study reveal that they are p-type polaronic insulators.

To explore the effect of cation size mismatch on activation energy, $E_{a}$ is plotted against cation size mismatch (Figure 10). From the plot, the inverse linear relation with $E_{a}$ and $\sigma^{2}$ is clear. The highest value of $E_{a}$ occurs at the lowest $\sigma^{2}$ and vice versa. As the cation size mismatch increases, activation energy decreases. It is well known that the electronic properties of perovskite oxides are often crucially connected with structural distortions around the Re site and/or transition metal sites [43, 44]. Linear fitting was performed to represent the variation of $E_{a}$ with $\sigma^{2}$ and the following relation was written from the fitting result:

$$
E_{a}\left(\sigma^{2}\right)=E_{a}(0)-m \sigma^{2}
$$

where $E_{a}(0)$ represents the activation energy at which $\sigma^{2}=0$, or the activation energy of the parent compound LFO which was not calculated in this study due to the compliance limit of the measuring devices, and $m$ is the slope of the best fitted line. Fitting results are: $E_{a}(0)=367.87 \pm 5.49 \mathrm{meV}$ and $m=934502.54 \mathrm{~K} / \AA^{2} . E_{a}(0)$ is the activation energy of the undoped sample LFO which was estimated with the use of (6) and the fitting results.

It is clearly evident (Figure 10) that room temperature resistivity decreases with increasing $\mathrm{Ca}$ and gets its minimum value at $10.47 \Omega \mathrm{cm}$ for sample LCFO30. As $\mathrm{Ca}^{2+}$ increases, the p-type carriers in a doped compound increase. This results to an increase in conductivity or equivalently a decrease in resistivity. Moreover, increasing p-type carriers causes $E_{a}$ to decrease, leading to a decrease in the energy gap of the doped compound. Furthermore, the decrease in resistivity possibly suggests that the Fermi level shifts to the nearer edge of the conduction band [45].

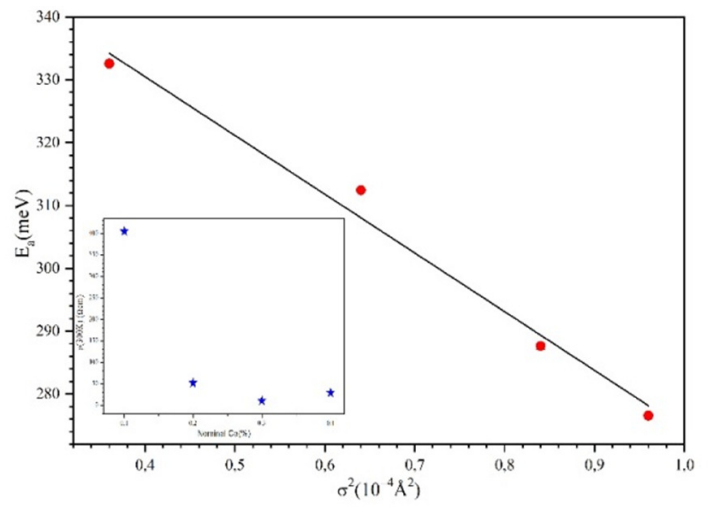

Fig. 10. Variation of $E a$ as a function of $\sigma^{2}$. Chance of $r_{0}$ at $300 \mathrm{~K}$ with nominal $\mathrm{Ca}$ (inset)

The room temperature resistivity of LCFO40 increased to a value of $29.47 \Omega \mathrm{cm}$. This may be the result of oxygen vacancy and appearance of impurity phases in that compound. Oxygen vacancies can provide electrons which compensate some of the holes created with $\mathrm{Ca}$ doping. This results to filling the $\mathrm{d}$ band of $\mathrm{Fe}^{4+}$ ions and transforming them to $\mathrm{Fe}^{3+}$. In addition, $\mathrm{Ca}$ deficiency and impurity phase $\mathrm{La}_{2} \mathrm{FeO}_{3}$ in LCFO40 leads to decrease of the $\mathrm{Fe}^{4+}$ ions. As a result, the number of holes contributing to conduction decreases and therefore resistivity increases.

\section{CONCLUSION}

The structural and electrical properties of nanosized $\mathrm{La}_{1}$ ${ }_{x} \mathrm{Ca}_{\mathrm{x}} \mathrm{FeO}_{3}$ compounds prepared via sol-gel method have been studied. Rietveld refinement of the XRD pattern of the samples indicates that all the samples have distorted orthorhombic structure with Pbnm space group, except the sample doped with $40 \% \mathrm{Ca}$ which is single phase. Increase of Ca doping leads to increase of the average distortion ratio. There is a drastic increase of average distortion ratio from parent compound LFO to LCFO10 and to LCFO20. Moreover, particle and grain growth are suppressed with $\mathrm{Ca}$. There is an inverse linear relation between unit cell volume and $\sigma^{2}$.

It was shown that electrical conduction in these compounds is thermally hooping of small polarons. The studied compounds are p-type polaronic insulators considering the conduction mechanism based on the values of $E_{a}$. As the $\mathrm{Ca}$ amount increases, $E_{a}$ and room temperature resistivity of the samples decreases. Inverse linear relation between $E_{a}$ with $\sigma^{2}$ and $T_{C O}$ with $\sigma^{2}$ were introduced. Moreover, charge ordering temperature, $T_{C O}$, decreases with increasing cation size mismatch. 


\section{ACKNOWLEDGMENT}

The author gratefully acknowledges the support of the Mugla Sitki Kocman University Scientific Research Projects Coordination Office (Grant No: 14/027).

\section{REFERENCES}

[1] K. Mukhopadhyay, A. S. Mahapatra, P. K. Chakrabarti, "Multiferroic behavior, enhanced magnetization and exchange bias effect of $\mathrm{Zn}$ substituted nanocrystalline $\mathrm{LaFeO}_{3}\left(\mathrm{La}_{(1-\mathrm{x})} \mathrm{Zn}_{\mathrm{x}} \mathrm{FeO}_{3}, \mathrm{x}=0.10\right.$, and 0.30$)$ ", Journal of Magnetism and Magnetic Materials, Vol. 329, pp. 133-141, 2013

[2] X Liu, B. Cheng, J. Hu, H. Qin, M. Jiang, "Preparation, structure, resistance and methane-gas sensing properties of nominal $\mathrm{La}_{1-\mathrm{x}} \mathrm{Mg}_{\mathrm{x}} \mathrm{FeO}_{3}$ ", Sensors and Actuators B: Chemical, Vol. 133, No. 1, pp. $340-344,2008$

[3] X. Dai, C. $\mathrm{Yu}$, Q. Wu, "Comparison of $\mathrm{LaFeO}_{3}, \mathrm{La}_{0.8} \mathrm{Sr}_{0.2} \mathrm{FeO}_{3}$, and $\mathrm{La}_{0.8} \mathrm{Sr}_{0.2} \mathrm{Fe}_{0.9} \mathrm{Co}_{0.1} \mathrm{O}_{3}$ perovskite oxides as oxygen carrier for partial oxidation of methane", Journal of Natural Gas Chemistry, Vol. 17, No. 4, pp. 415-418, 2008

[4] Q. Lin, J. Xu, F. Yang, X. Yang, Y. He, "The influence of Ca substitution on $\mathrm{LaFeO}_{3}$ nanoparticles in terms of structural and magnetic properties", Journal of Applied Biomaterials and Functional Materials, Vol. 16, No. 1, pp. 17-25, 2018

[5] A. M. Stoneham, J. Gavartin, A. L. Shluger, A. V. Kimmel, D. M. Ramo, H. M. Ronnow, G. Aeppli, C. Renner, "Trapping, self-trapping and the polaron family", Journal of Physics: Condensed Matter, Vol. 19, No. 25, Article ID 255208, 2007

[6] Z. Zhou, L. Guo, H. Yang, Q. Liu, F. Ye, "Hydrothermal synthesis and magnetic properties of multiferroic rare-earth orthoferrites", Journal of Alloys and Compounds, Vol. 583, pp. 21-31, 2014

[7] L. Hou, G. Sun, K. Liu, Y. Li, F. Gao, "Preparation, characterization and investigation of catalytic activity of Li-doped $\mathrm{LaFeO}_{3}$ nanoparticles", Journal of Sol-Gel Science and Technology, Vol. 40, pp. 9-14, 2006

[8] K. Taniguchi, N. Okinaka, T. Akiyama, "Preparation and characterization of $\mathrm{La}_{1-x} \mathrm{~K}_{x} \mathrm{FeO}_{3}(x=0-1)$ by self-propagating hightemperature synthesis for use as soot combustion catalyst", Journal of Alloys and Compounds, Vol. 509, No. 10, pp. 4084-4088, 2011

[9] L. Sun, H. Qin, K. Wang, M. Zhao, J. Hu, "Structure and electrical properties of nanocrystalline $\mathrm{La}_{1-x} \mathrm{Ba}_{x} \mathrm{FeO}_{3}$ for gas sensing application", Materials Chemistry and Physics, Vol. 125, No. 1-2, pp. 305-308, 2011

[10] P. Song, H. Qin, L. Zhang, K. An, Z. Lin, J. Hu, M. Jiang, "The structure, electrical and ethanol-sensing properties of $\mathrm{La}_{1-x} \mathrm{~Pb}_{x} \mathrm{FeO}_{3}$ perovskite ceramics with $x \leq 0.3$ ", Sensors and Actuators B: Chemical, Vol. 104, No. 2, pp. 312-316, 2005

[11] R. Andoulsi, K. H. Naifer, M. Ferid, "Structural and electrical properties of calcium substituted lanthanum ferrite powders", Powder Technology, Vol. 230, pp. 183-187, 2012

[12] L. B. Kong, Y. S. Shen, "Gas-sensing property and mechanism of $\mathrm{Ca}_{\mathrm{x}} \mathrm{La}_{1 \square \mathrm{x}} \mathrm{FeO}_{3}$ ceramics”, Sensors and Actuators B: Chemical, Vol. 30, No. 3, pp. 217-221, 1996

[13] M. H. Hung, M. V. M. Rao, D. S. Tsai, "Microstructures and electrical properties of calcium substituted $\mathrm{LaFeO}_{3}$ as SOFC cathode", Materials Chemistry and Physics, Vol. 101, No. 2-3, pp. 297-302, 2007

[14] A. Benali, S. Azizi, M. Bejar, E. Dhahri, M. F. P. Graca, "Structural, electrical and ethanol sensing properties of double-doping $\mathrm{LaFeO}_{3}$ perovskite oxides", Ceramics International, Vol. 40, No. 9, pp. 1436714373,2014

[15] L. Zhang, J. Hu, P. Song, H. Qin, M. Jiang, "Electrical properties and ethanol-sensing characteristics of perovskite $\mathrm{La}_{1-x} \mathrm{~Pb}_{x} \mathrm{FeO}_{3}$ ", Sensors and Actuators B: Chemical, Vol. 114, No. 2, pp. 836-840, 2006

[16] C. Shi, H. Qin, M. Zhao, X. Wang, L. Li, J. Hu, "Investigation on electrical transport, $\mathrm{CO}$ sensing characteristics and mechanism for nanocrystalline $\mathrm{La}_{1-x} \mathrm{Ca}_{x} \mathrm{FeO}_{3}$ sensors", Sensors and Actuators B: Chemical, Vol. 190, pp. 25-31, 2014
[17] R. Andoulsi, K. H. Naifer, M. Ferid, "Electrical conductivity of $\mathrm{La}_{1-x} \mathrm{Ca}_{x} \mathrm{FeO}_{3-\delta}$ solid solutions", Ceramics International, Vol. 39, No. 6, pp. 6527-6531, 2013

[18] A. E. Irmak, E. Tasarkuyu, A. Coskun, M. Acet, Y. Samancioglu, S. Akturk, "Magnetic and electrical transport properties of $\mathrm{La}_{0.65} \mathrm{Ca}_{0.30} \mathrm{~Pb}_{0.05} \mathrm{Mn}_{0.90} \mathrm{Cu}_{0.10} \mathrm{O}_{3}$ compounds: Thermal hysteresis", Physica B: Condensed Matter, Vol. 470-471, pp. 56-63, 2015

[19] J. R. Carvajal, "Fullprof: A program for rietveld refinement and pattern matching analysis", Satellite Meeting on Powder Diffraction of the XV Congress of the IUCr, Toulouse, France, July 19-8, 1990

[20] J. $\mathrm{Li}$, "Investigation of orthorhombic perovskite $\mathrm{La}_{1-} x \mathrm{Ca} \mathrm{FeO} \mathrm{F}_{3-\mathrm{y}}(0 \leq x \leq$ 0.50)", Physica Scripta, Vol. 45, pp. 62-64, 1992

[21] A. L. Patterson, "The Scherrer formula for X-ray particle size determination”, Physical Review, Vol. 56, pp. 978-982, 1939

[22] K. Ellmer, A. Bikowski, "Intrinsic and extrinsic doping of $\mathrm{ZnO}$ and $\mathrm{ZnO}$ alloys", Journal of Physics D: Applied Physics, Vol. 49, No. 41, Article ID 413002, 2016

[23] Z. Jirak, S. Krupicka, Z. Simsa, M. Dlouha, S. Vratislav, "Neutron diffraction study of $\mathrm{Pr}_{1-x} \mathrm{Ca}_{x} \mathrm{MnO}_{3}$ perovskites", Journal of Magnetism and Magnetic Materials, Vol. 53, No. 1-2, pp. 153-166, 1985

[24] D. Nath, F. Singh, R. Das, "X-ray diffraction analysis by WilliamsonHall, Halder-Wagner and size-strain plot methods of CdSe nanoparticles-a comparative study", Materials Chemistry and Physics, Vol. 239, Article ID 122021, 2020

[25] Y. F. Sun, S. B. Liu, F. L. Meng, J. Y. Liu, Z. Jin, L. T. Kong, J. H. Liu, "Metal oxide nanostructures and their gas sensing properties: A review", Sensors, Vol. 12, No. 3, pp 2610-2631, 2012

[26] G. A. Gamal, F. A. A. Mufadi, A. H. Said, "Effect of iron additives on the microstructure of hydroxyapatite", Engineering, Technology \& Applied Science Research, Vol. 3, No. 6, pp. 532-539, 2013

[27] M. F. Garcia, J. A. Rodriguez, "Metal oxide nanoparticles", in: Encyclopedia of Inorganic and Bioinorganic Chemistry, John Wiley \& Sons, 2011

[28] S. Boumous, S. Belkhiat, F. Kharchouche, "MgO effect on the dielectric properties of $\mathrm{BaTiO}_{3}$ ", Engineering, Technology \& Applied Science Research, Vol. 9, No. 3, pp. 4092-4099, 2019

[29] V. Rathod, A. V. Anupama, V. M. Jali, V. A. Hiremath, B. Sahoo, "Combustion synthesis, structure and magnetic properties of $\mathrm{Li}-\mathrm{Zn}$ ferrite ceramic powders", Ceramics International, Vol. 43, No. 16, pp. 14431-14440, 2017

[30] M. Allieta, M. Scavini, L. L. Presti, M. Coduri, L. Loconte, S. Cappelli, C. Oliva, P. Ghigna, P. Pattison, V. Scagnoli, "Charge ordering transition in GdBaCo2O5: Evidence of reentrant behavior", Physical Review B, Vol. 88, Article ID 214104, 2013

[31] Y. Wang, Y. Sui, X. Wang, W. Su, "Structure, transport and magnetic properties of electron-doped perovskites $\mathrm{R}_{\mathrm{x}} \mathrm{Ca}_{1-\mathrm{x}} \mathrm{MnO}_{3}(\mathrm{R}=\mathrm{La}, \mathrm{Y}$ and Ce)", Journal of Physics: Condensed Matter, Vol. 21, Article ID 196004, 2009

[32] M. Respaud, J. M. Broto, H. Rakoto, J. Vanacken, P. Wagner, C. Martin, A. Maignan, B. Raveau, "H-T magnetic phase diagrams of electrondoped $\mathrm{Sm}_{1-\mathrm{x}} \mathrm{Ca}_{\mathrm{x}} \mathrm{MnO}_{3}$ : Evidence for phase separation and metamagnetic transitions", Physical Revew B, Vol. 63, Article ID 144426, 2001

[33] L. M. R. Martinez, J. P. Attfield, "Cation disorder and size effects in magnetoresistive manganese oxide perovskites", Physical Review B, Vol. 54, Article ID R15622, 1996

[34] A. Arulraj, P. N. Santhosh, R. S. Gopalan, A. Guha, A. K. Raychaudhuri, N. Kumar, C. N. R. Rao, "Charge ordering in the rareearth manganates: The origin of the extraordinary sensitivity to the average radius of the A-site cations, $\left\langle\mathrm{r}_{\mathrm{A}}>\right.$ ", Journal of Physics: Condensed Matter, Vol. 10, Article ID 8497, 1998

[35] A. J. Millis, P. B. Littlewood, B. I. Shraiman, "Double exchange alone does not explain the resistivity of $\mathrm{La}_{1-\mathrm{x}} \mathrm{SrxMnO}_{3}$ ”, Physical Review Letters, Vol. 74, Article ID 5144, 1995

[36] W. H Jung, E. Icuchi, "Transition from hopping conduction to band conduction in $\mathrm{LaFe}_{x} \mathrm{Ni}_{1-x} \mathrm{O}_{3}$ ", Philosophical Magazine B, Vol. 73, pp. $873-891,1996$ 
[37] N. F. Mott, E. A. Davis, Electronics processes in non-crystalline materials, Oxford University Press, 1971

[38] I. G Austin, N. F. Mott, "Polarons in crystalline and non-crystalline materials", Advances in Physics, Vol. 18, No. 71, pp. 41-102, 1969

[39] S. Brahma, R. N. P. Choudhary, A. K. Thakur, "AC impedance analysis of $\mathrm{LaLiMo}_{2} \mathrm{O}_{8}$ electroceramics", Physica B: Condensed Matter, Vol. 355, No. 1-4, pp. 188-201, 2005

[40] P. Ganguly, A. K. Jha, K. L. Deori, "Complex impedance studies of tungsten-bronze structured $\mathrm{Ba}_{5} \mathrm{SmTi}_{3} \mathrm{Nb}_{7} \mathrm{O}_{30}$ ferroelectric ceramics", Solid State Communications, Vol. 146, No. 11-12, pp. 472-477, 2008

[41] M. Idrees, M. Nadeem, M. M. Hassan, "Investigation of conduction and relaxation phenomena in $\mathrm{LaFe}_{0.9} \mathrm{Ni}_{0.1} \mathrm{O}_{3}$ by impedance spectroscopy", Journal of Physics D: Applied Physics, Vol. 43, No. 15, Article ID 155401,2010

[42] H. Rahmouni, R. Jemai, M. Nouiri, N. Kallel, F. Rziguac, A. Selmi, K. Khirouni, S. Alaya, "Admittance spectroscopy and complex impedance analysis of Ti-modified $\mathrm{La}_{0.7} \mathrm{Sr}_{0.3} \mathrm{MnO}_{3}$ ", Journal of Crystal Growth, Vol. 310, No. 3, pp. 556-561, 2008

[43] S. Satpathy, Z. S. Popovic, F. R. Vukajlovic, "Electronic structure of the perovskite oxides: $\mathrm{La}_{1-\mathrm{x}} \mathrm{Ca}_{\mathrm{x}} \mathrm{MnO}_{3}$ ", Physical Review Letters, Vol. 76 , Article ID 960, 1996

[44] W. Khan, A. H. Naqvi, M. Gupta, S. Husain, R. Kumar, "Small polaron hopping conduction mechanism in Fe doped $\mathrm{LaMnO}_{3}$ ", The Journal of Chemical Physics, Vol. 135, Article ID 054501, 2011

[45] Y. Liu, X. Y. Qin, "Temperature dependence of electrical resistivity for Ca-doped perovskite-type $\mathrm{Y}_{1-x} \mathrm{Ca}_{x} \mathrm{CoO}_{3}$ prepared by sol-gel process", Journal of Physical Chemistry of Solids, Vol. 67, No. 8, pp. 1893-1898, 2006 